RESEARCH ARTICLE

\title{
An Exploratory Study of the Knowledge, Perceptions, Confidence Levels, and Practices of Oral Implantology
}

\author{
Jana M Pierce ${ }^{1 *}$, JoAnn R Gurenlian ${ }^{2}$ and Jacque Freudentha/ ${ }^{3}$ \\ ${ }^{1}$ Instructor and Program Coordinator, Department of Health Sciences/Dental Assisting, College of Marin, USA \\ ${ }^{2}$ Professor and Graduate Program Director, Department of Dental Hygiene, Idaho State University, USA \\ ${ }^{3}$ Associate Professor and Chair, Department of Dental Hygiene, Idaho State University, USA
}

*Corresponding author: Jana M Pierce, CDA, RDH, MS, PhD student, Capella University, 2117 Dellwood Ave Medford, OR 97504, USA, Tel: 530-921-0122

\begin{abstract}
Background: The All-On-4 (AO4) oral implant process positively impacts oral health related quality of life for edentulous individuals. The purpose of this study was to identify the knowledge level, perceptions, confidence levels, and self-reported practices of oral professionals regarding $\mathrm{AO} 4$.

Methods: A convenience sample of 324 dentists and 237 dental hygienists licensed in the state of Oregon were invited to participate in a self-administered electronic questionnaire. Data were analyzed using frequencies, percentages, and chi-square test.

Results: The study yielded a $15.33 \%$ response rate. Forty-nine respondents were dentists (15.17\%) and 35 were dental hygienists (15.56\%). Most dentists were male. Most dental hygienists were female. The majority of respondents indicated practicing in the dental field for over 30 years with minimal completion of specialty education. Few respondents indicated completing a case or participating in maintenance therapy for $\mathrm{AO} 4$. The majority of respondents felt their $\mathrm{AO} 4$ professional training did not prepare them to offer $\mathrm{AO} 4$ to patients. The majority of participants $(65.6 \%$ of dentists and $83.3 \%$ of dental hygienists) indicated a need for further $\mathrm{AO} 4$ training. Confidence levels were disproportionate to the knowledge level, education, and experience of the participants.

Conclusion: Success rates for complex implant therapies can be attained if knowledge and clinical expertise of providers are congruent. Supplemental studies designed to compare the content of complex oral implant education received to the clinical expertise of the provider would aid in determination of the potential for restructure, elimination, or addition of $\mathrm{AO} 4$ to continuing education and/or containment to advanced education.
\end{abstract}

\section{Keywords}

Oral implantology, AO4, Fixed dental prosthesis, Oral implant education, Dental hygienist's role, Oral health-related quality of life (OHrQoL)

\begin{abstract}
Abbreviations
OHrQoL: Oral Health-Related Quality of Life; OHIP: Oral Health Impact Profile; FDPs: Fixed Dental Prostheses; M-RPDs: Metal-Based Removable Partial Dentures; ADs: Acrylic Removable Partial Dentures; CDs: Complete Dentures; AO4: All-On-Four; CBCT: Cone-Beam Computed Tomography Scans; CVI: Content Validity Index; PIMT: Peri-Implant Maintenance Therapy; CE: Continuing Education; CODA: Commission on Dental Accreditation
\end{abstract}

\section{Introduction}

Approximately one quarter of the U.S. population is over the age of 55, representing a demographic that is living longer, experiencing more chronic degenerative diseases, and taking more prescription medications than past generations [1,2]. When compared to the $79 \%$ increase in the number of adults projected to be in the aging population, the number of adults in need of one or two complete dentures is expected to increase to a projected 37.9 million in 2020 [3]. This population shift is anticipated to cause a substantial spike in individuals seeking oral implant and prosthetic therapy, increasing the need for treatment modalities that can augment occlusal function and increase oral health-related quality of life (OHrQoL) [4-8].

Oral function and implant therapy are interrelated. Data obtained through a large-scale cross-sectional survey of 617 elderly individuals living in long-term care facilities led to the rating of occlusal status as a significant risk factor for malnutrition among the geriatric population [9]. Oral function is linked as a co-variable in food

Citation: Pierce JM, Gurenlian JR, Freudenthal J (2018) An Exploratory Study of the Knowledge, Perceptions, Confidence Levels, and Practices of Oral Implantology. Int J Oral Dent Health 4:068. doi. org/10.23937/2469-5734/1510068

Accepted: November 01, 2018: Published: November 03, 2018

Copyright: (C) 2018 Pierce JM, et al. This is an open-access article distributed under the terms of the Creative Commons Attribution License, which permits unrestricted use, distribution, and reproduction in any medium, provided the original author and source are credited. 
selection, avoidance, and consumption in older adults $[10,11]$. Implant supported fixed dental prosthesis include overdentures and more innovative developments such as full arch immediate load oral implant processes $[12,13]$. These technologies can increase OHrQoL by improving occlusal function, which, in turn, facilitate eating and promote adequate nutrition intake $[6,10]$.

Locker's conceptual model and a frequency based 14 item abbreviated index oral health impact profile (OHIP) was used to compare fixed versus removable denture outcomes [6]. Previous studies conducted on the subject of prosthetic therapy compared over-dentures to removable dentures only. The researchers who developed the OHIP noted that the study was the "first investigation that to evaluate(s) the self-rated satisfaction of patients treated with fixed dental prostheses (FDPs), metal-based removable partial dentures (M-RPDs), acrylic removable partial dentures (ADs), and complete dentures (CDs)" [6]. A consecutive sampling of subjects seeking prosthetic rehabilitation were recruited and compared to the sample population (P-population; $n$ = 123). The Fichner Index for evaluation of prosthetic function/occlusion was applied to the sample P-population of 123 Spanish adults wearing conventional dental prostheses and not currently seeking dental treatment. The results of the study revealed that subjects receiving ADs or CDs reported lower OHrQoL scores than subjects requiring M-RPDs or FDPs. While subjects reported overall satisfaction and generalized well-being associated with conventional prosthetic therapy, 20 percent of the study population reported discomfort and chewing dysfunction. Fixed dental prosthesis therapy appeared to be the most significant contributing factor in eating facilitation for the study subjects [6].

For a quarter of a century, studies of this nature guided the progressive evolution of oral implantology and a collaborative approach to patient treatment emerged [5]. Treatment options advanced from single tooth implants to full arch immediate loaded implant prosthesis overdenture alternatives. Advancement in design and technique can now provide 100 percent restoration of occlusal function through a fixed retrievable prosthesis immediate load oral implant process known as the All-On-Four (AO4; All-on- $4^{\circledR}$; Nobel Biocare Services AG, Zurich, Sweden) process [13]. The AO4 process is a sequential phasic multifaceted procedure involving state of the art digitized technology that requires the interprofessional collaboration of all providers involved (dental hygienist, dentist, implantologist, and medical professional) [13-18]. The procedure provides for immediate occlusal function for the patient, and a level of instant gratification, both visually and phonetically, not achieved by past fixed prosthesis treatment processes. The AO4 process has a cumulative success rate of 99.6 percent with a 100 percent definitive survival rate for the prosthetic $[16,18]$.

The pioneer for the AO4 process was Paulo Malo [19]. Malo identified edentulism as a progressive problem and developed the AO4 standard protocol in 1993, as part of a pilot study on oral implants [19]. Over the next 24 years Malo and colleagues, fostered studies and publications that revolutionized the oral implant fixed prosthesis process. Figure 1 highlights a timeline of the evolution and research of the $\mathrm{AO} 4$ process and Figure 2 illustrates radiographic depiction of the evolved procedure to date. Malo found the AO4 process to be a predictable and repeatable protocol when three conditions were met: The risk assessment was comprehensive, the patient was selected within the recommended guidelines, and the NobelGuide (NobelGuide ${ }^{\circledR}$; Nobel Biocare Services AG, Zurich, Sweden) protocol was followed [4,19-23].

A review of literature revealed parallel success of multiple studies conducted over the past 15 years, by researchers independent of Malo, which supported the efficacy of immediate loaded fixed implant prosthesis

\section{Evolution of the All-on-4® Process} The Studies of Paulo Malo

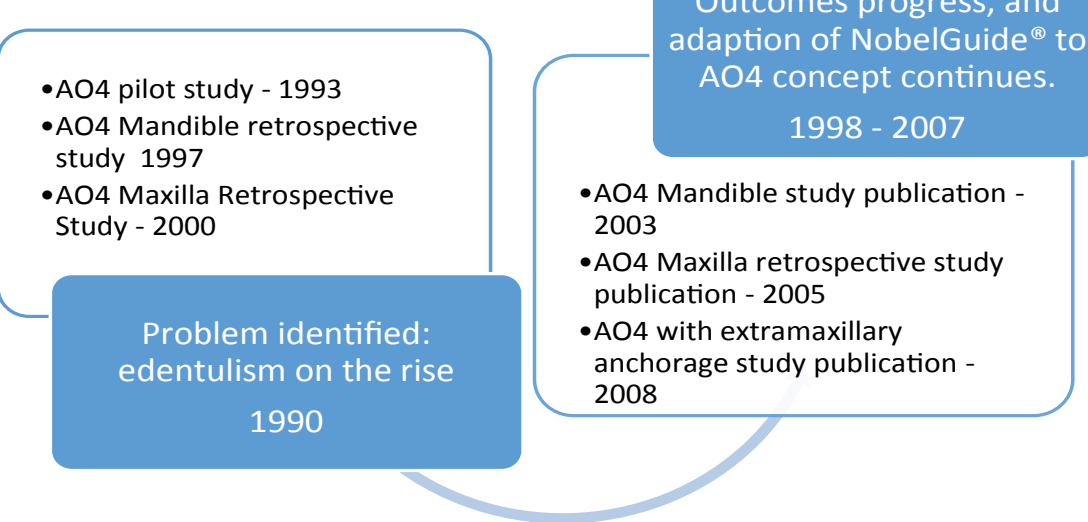

Outcomes progress, and daption of NobelGuide ${ }^{\circledR}$ to AO4 concept continues. $1998-2007$

-AO4 Mandible study publication 2003

-AO4 Maxilla retrospective study publication - 2005

with extramaxillary nchorage study publication 2008

Figure 1: Evolution timeline of the All-on- $4^{\circledR}$ process, developed from the studies of Paulo malo (1993-2014).
-AO4 Mandible 10-year followup and Maxilla 5-year followup study publication - 2011 -AO4 Maillary anchorage 3 year follow-up study - 2012

-A04 mandible retrospective 7year follow-up study - 2014

AO4 accepted as a viable and predicable treatment option with $100 \%$ restoration of occlusal function. 2011 


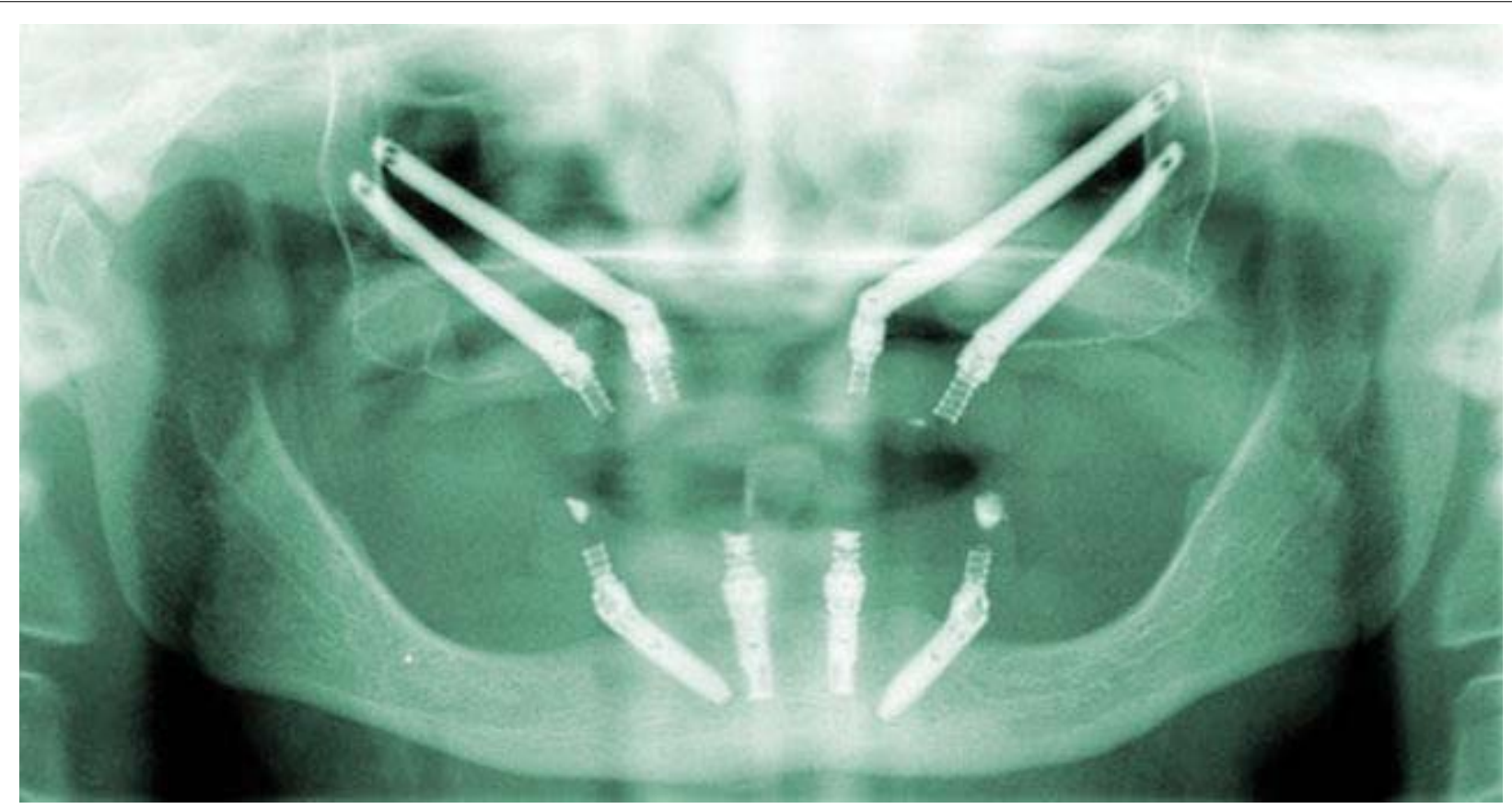

Figure 2: All-on- ${ }^{\circledR}$ fixed implant prosthesis radiograph (current treatment technique).

processes. Progressively, the studies consistently; a) Confirmed rehabilitation with prefabricated fixed prosthesis supported by dental implants placed in accordance to AO4 provisional clinical protocol was a feasible and predictable treatment option, and b) Demonstrated between 97.9 percent and 100 percent implant survival rate (one to 15-year follow-up) [4,13,14,19,20,22-26]. As a whole, overall patient satisfaction for participants was reported to be high and OHrQoL was improved due to increased occlusal function [25]. Based on multiple studies and refinement of the process, with parallel initiation of Nobel Guide protocol, digitized software, and cone-beam computed tomography scans (CBCT), the AO4 process revolutionized current implantology protocols $[4,13,14,19,20,22-26]$.

The AO4 concept involves the placement of four dental implants, with two implants placed axially and two angled, supporting a provisional fixed immediately loaded full-arch prosthesis using CAD/CAM technology (Figure 2). [13,25,26]. In most cases the dental implants are placed using a minimally invasive flapless, mini-flap, or flap surgical access approach. The innovation behind the AO4 process is the angled posterior implants, which allow for reduced need of vertical bone augmentation and consequent decreased treatment time and increased healing time. Following the laboratory and surgical template construction, the $\mathrm{AO} 4$ procedure is performed in one surgical appointment with surgery times varying on a case-by-case basis $[25,26]$. This streamlined approach allows for immediate loading of the prosthesis, in most cases the same day as treatment, providing the patient with immediate occlusal function [27].

\section{Objectives}

The advancement of the AO4 process has evolved to meet the increased elderly population and the corre- sponding demand of oral implants and fixed prosthesis as an option for tooth replacement. The introduction of this process dictates the need for maintenance and routine clinical assessments, by the entire oral implant team, of the long-term stability of the implants and prosthesis placed [28-32]. Although research exists to support the efficacy of the $\mathrm{AO} 4$ process, there is a gap in the literature concerning whether dental professionals are equipped, proficient, and prepared to present and provide advanced oral implant therapy and processes such as the AO4 treatment option to their patients. Therefore, the purpose of this study was to discover the knowledge, perceptions, confidence levels, and self-reported practices of dentists and dental hygienists regarding the $\mathrm{AO} 4$ immediate load fixed prosthesis oral implant process and determine if any statistically significant differences or relationships existed within and between the study groups.

The following null hypotheses guided the conduct of this study 1) There is no statistically significant difference between dentist's and dental hygienist's knowledge level, perceptions, self-reported practices, and confidence levels regarding the $\mathrm{AO} 4$ immediate load oral implant process, 2) There is no statistically significant difference between age, sex, and years of practice, and dentists and dental hygienists knowledge level, perceptions, self-reported practices, and confidence levels regarding the AO4 immediate load oral implant process, and 3) There is no statistically significant relationship between the education level of dentists and dental hygienists and their self-reported practices, knowledge, perceptions, and confidence levels about the AO4 immediate load oral implant process.

\section{Methods}

The research method for this exploratory study was 
a self-designed 35-item survey questionnaire of dentists and dental hygienists. The study was completed online by a convenience sample of licensed dentists $(N=323)$ and dental hygienists $(N=225)$ practicing in the state of Oregon. The Oregon Board of Dentistry provided the list of dentists and dental hygienists who had publicly accessible email addresses [33].

The study protocol was submitted for expedited review and approved by the Human Subjects Committee (Approval \# MS 8048). An introduction letter was emailed to the individuals explaining the purpose of the study, followed by the survey letter including informed consent and the survey link. Four letters in total were emailed to encourage participation prior to the close of the study.

The instrument addressed the $\mathrm{AO} 4$ assessment process, current practices regarding the $\mathrm{AO} 4$ process, demographics, experience with the $\mathrm{AO} 4$ process, self-reported outcome of the $\mathrm{AO} 4$ process, education relating to the $\mathrm{AO} 4$ process, and preference for future advanced implant and $\mathrm{AO} 4$ process education. The primary focus of the study related predominately to the $\mathrm{AO} 4$ assessment process and the knowledge and professional education of the participant. Instrument validity was assessed using a six-expert panel and a content validity index (CVI). The CVI experts were included based on their experience with oral implantology and the $\mathrm{AO} 4$ process.
The panel consisted of two oral surgeons, two dentists, and two dental hygienists. The parameters for the CVI scale were constructed from the findings of Polit and Beck [34]. In addition, the survey was tested for reliability through a test/re-test method. This testing involved eight licensed oral practitioners in the state of Oregon, four dentists and four dental hygienists. Feedback from the CVI and reliability testing were incorporated as appropriate. Findings showed the survey to be highly valid and reliable.

Results were summarized in descriptive form using frequencies and percentages. A chi-square test was used for each null hypothesis. The level of significance was established at $p \leq 0.05$.

\section{Results}

A total of 548 surveys were emailed to licensed Oregon dental practitioners. Of this group, 84 participants completed the survey during the spring of 2016, yielding a response rate of $15.33 \%$. Of the respondents, 49 were dentists $(15.17 \%)$ and 35 were dental hygienists (15.56\%) (Table 1). highlights the demographic data and AO4 training for the dentists and dental hygienists who participated in the study. The majority of dentists were male, and the majority of dental hygienists were female. Age ranges differed for the groups. The majority of dentists were in the age range of $41-50$ years of age with the

Table 1: Demographic data and AO4 training of dentists and dental hygienists.

\begin{tabular}{|c|c|c|c|c|c|}
\hline \multirow{2}{*}{\begin{tabular}{|l|} 
Dentists \\
Characteristic \\
\end{tabular}} & & \multirow[b]{3}{*}{$\%$} & \multicolumn{3}{|l|}{ Dental hygienists } \\
\hline & \multirow[b]{2}{*}{$\mathbf{N}$} & & \multirow[b]{2}{*}{ Gender } & \multirow[b]{2}{*}{$\mathbf{N}$} & \multirow[b]{2}{*}{$\%$} \\
\hline Gender & & & & & \\
\hline Male & 39 & 79.6 & Male & 1 & 2.9 \\
\hline Female & 10 & 23.4 & Female & 34 & 97.1 \\
\hline Degree level & $\mathbf{N}$ & $\%$ & Degree level $^{\mathrm{a}}$ & $\mathbf{N}$ & $\%$ \\
\hline DDS or DMD & 44 & 89.8 & $\mathrm{RDH}$ or $\mathrm{LDH}$ & 12 & 34.3 \\
\hline DDS or DMD + MS & 5 & 10.2 & $\mathrm{RDH}$ or $\mathrm{LDH}+\mathrm{BS}$ & 15 & 42.9 \\
\hline DDS or DMD + PhD & - & - & $\mathrm{RDH}$ or $\mathrm{LDH}+\mathrm{MS}$ & 7 & 20 \\
\hline Periodontist & 2 & 4.1 & $\mathrm{RDH}$ or $\mathrm{LDH}+\mathrm{PhD}$ & 2 & 5.7 \\
\hline Orthodontist & 3 & 6.1 & $\begin{array}{l}\text { RDH or } \mathrm{LDH}+\text { Advanced (ex = } \\
\text { ADT) }\end{array}$ & 1 & 2.9 \\
\hline Aesthetic dentist & 1 & 2 & Endorsement or permit & & \\
\hline Pediatric dentist & 1 & 2 & Restorative & 7 & 20 \\
\hline Oral surgeon & 1 & 2 & Limited access (LAP) & 9 & 25.7 \\
\hline Medical degree & 1 & 2 & Nitrous oxide sedation & 31 & 88.6 \\
\hline Oral sedation license & 11 & 22.4 & Anesthesia & 32 & 91.4 \\
\hline Other-unspecified & 7 & 14.3 & & & \\
\hline Total & 49 & 100 & Total & 35 & 100 \\
\hline Age range (year) & \multicolumn{2}{|c|}{ Dentists } & \multicolumn{3}{|l|}{ Dental hygienists } \\
\hline Characteristic & $\mathbf{N}$ & $\%$ & $\mathbf{N}$ & \multicolumn{2}{|l|}{$\%$} \\
\hline $18-30$ & 1 & 2.0 & 2 & \multicolumn{2}{|l|}{5.7} \\
\hline $31-40$ & 7 & 14.3 & 4 & \multicolumn{2}{|l|}{11.4} \\
\hline $41-50$ & 14 & 28.6 & 7 & \multicolumn{2}{|c|}{20.0} \\
\hline $51-60$ & 11 & 22.4 & 8 & \multicolumn{2}{|c|}{22.9} \\
\hline
\end{tabular}




\begin{tabular}{|c|c|c|c|c|}
\hline $61-70$ & 11 & 22.4 & 11 & 31.4 \\
\hline $71+$ & 5 & 10.2 & 3 & 8.6 \\
\hline AO4 Training ${ }^{b}$ & $\mathbf{N}$ & $\%$ & $\mathbf{N}$ & $\%$ \\
\hline Received AO4 training & 13 & 26 & 1 & 2.9 \\
\hline Did not receive AO4 training & 37 & 74 & 34 & 97.1 \\
\hline Years of practice & $\mathbf{N}$ & $\%$ & $\mathbf{N}$ & $\%$ \\
\hline $1-5$ & 3 & 6.1 & 2 & 5.7 \\
\hline $6-10$ & 6 & 12.2 & 2 & 5.7 \\
\hline $11-15$ & 4 & 8.2 & 2 & 5.7 \\
\hline $16-20$ & 9 & 18.4 & 5 & 14.3 \\
\hline $21-30$ & 10 & 20.4 & 9 & 25.7 \\
\hline $31+$ & 17 & 34.7 & 15 & 42.9 \\
\hline Field of practice & $\mathbf{N}$ & $\%$ & $\mathbf{N}$ & $\%$ \\
\hline General/family dentistry & 37 & 75.5 & 19 & 54.3 \\
\hline Periodontics & 2 & 4.1 & 2 & 5.7 \\
\hline Oral surgery & 2 & 4.1 & 1 & 2.9 \\
\hline Pediatrics & 1 & 2 & 1 & 2.9 \\
\hline Other-unspecified & 7 & 14.3 & 12 & 34.3 \\
\hline Total & 49 & 100 & 35 & 100 \\
\hline
\end{tabular}

andividuals could select more than one response; ${ }^{\mathrm{b}}$ For those who received $\mathrm{AO} 4$ training, 13 participants received their training in the form of continuing education; 6 participants (46.2\%) received instruction through a speaker forum with class interaction; 7 participants $(38.5 \%)$ received instruction through a hands-on course over a period of days/more than one day.

Table 2: Dentists and dental hygienists confidence levels regarding AO4.

\section{Dentists}

\section{Characteristic}

How would you describe your level of understanding of the AO4 process?

Know nothing about the AO4 process.

\begin{tabular}{|l|l|l|l|l|}
\hline \multicolumn{5}{|c|}{ Nental hygienist } \\
\hline $\mathbf{N}$ & $\%$ & $\mathbf{N}$ & $\%$ \\
\hline 8 & 24.2 & 16 & 72.7 \\
\hline 3 & 9.1 & 4 & 18.2 \\
\hline 8 & 24.2 & 1 & 4.5 \\
\hline 13 & 39.4 & 1 & 4.5 \\
\hline 1 & 3.0 & 0 & 0 \\
\hline 33 & 100 & 22 & 100 \\
\hline
\end{tabular}

appropriate case.

Know of the AO4 Process and how to recognize a patient for a case, but do not feel confident enough to present AO4 as an option to my patients.

Well versed in $\mathrm{AO} 4$ process and feel comfortable presenting $\mathrm{AO} 4$ as an option to patients but 13 would like to know more.

Consider themselves an expert in $\mathrm{AO} 4$ process

Total 33 with $\mathrm{AO4}$

To what

Somewhat

Confident

Expert

\begin{tabular}{|l|l|l|l|}
\hline 7 & 24.1 & 12 & 70.6 \\
\hline 7 & 24.1 & 1 & 5.9 \\
\hline 13 & 44.8 & 4 & 23.5 \\
\hline 2 & 6.9 & 0 & 0 \\
\hline 29 & 100 & 18 & 100 \\
\hline
\end{tabular}

Total

How would you rate your confidence level with regard to the AO4 Process? - Only respondents who had participated in an AO4 case answered this question.

Extremely confident

Moderately confident

Slightly confident

Not confident

Total

\begin{tabular}{|l|l|l|l|}
\hline 2 & 8.7 & - & - \\
\hline 10 & 43.5 & 1 & 100 \\
\hline 3 & 13.0 & - & - \\
\hline 8 & 34.8 & - & - \\
\hline 23 & 100 & 1 & 100 \\
\hline
\end{tabular}

majority of dental hygienists between the ages of 61-70 years of age. Most dentists and dental hygienists had been practicing for over 30 years in a general dentistry practice and the majority of respondents indicated they had not received training regarding the $\mathrm{AO} 4$ process. Pearson's chi-square test was used to determine the significant difference found between the genders of the oral professionals participating in this study $\left(\mathrm{X}^{2}=\right.$ 48.196, $d f=1, p<0.001)$. There was no significant difference in age range when comparing dentists to dental hygienists $\left(X^{2}=2.186, d f=5, p=0.823\right)$. A marginal number of dentists (17.8\%) indicated they had completed an AO4 case. One dental hygienist (3.0\%) indicated completion of a case. Approximately 20 percent of dentists 
considered their $\mathrm{AO} 4$ cases successful. A majority of dentists (64.7\%) and dental hygienists (87.5\%) felt their $\mathrm{AO} 4$ professional training did not prepare them to offer the $\mathrm{AO} 4$ process to their patients. A similar number of participants ( $65.6 \%$ of dentists and $83.3 \%$ of dental hygienists) indicated a need for further $\mathrm{AO} 4$ training.

Confidence levels regarding the $\mathrm{AO} 4$ process are summarized in (Table 2). When questioned regarding their level of understanding of the AO4 process, 39.4\% of dentists felt they were well versed in the $\mathrm{AO} 4$ process and the majority of dental hygienists $(75 \%)$ indicated they knew nothing about the $\mathrm{AO} 4$ process. Approximately one half $(51.7 \%)$ of dentists felt comfortable providing maintenance therapy to $\mathrm{AO} 4$ patients, while the majority of dental hygienists $(76.5 \%)$ did not feel comfortable with provision of maintenance therapy to
AO4 patients. Almost half of the dentists $(44.8 \%)$ and one quarter of the dental hygienists (23.5\%) who had participated in an $\mathrm{AO} 4$ case felt moderately confident with the process. The vast majority of both dentists $(62.9 \%)$ and dental hygienists $(87.5 \%)$ indicated they had not completed an $\mathrm{AO} 4$ case. Half of the dentists who had completed an $\mathrm{AO} 4$ case indicated participation in the $\mathrm{AO} 4$ process as a provider and half indicated participating as an observer, while the majority of dental hygienists (4 out of 5) participated as an observer only in the $\mathrm{AO} 4$ process.

Table 3 summarizes the knowledge of the study participants with relation to the $\mathrm{AO} 4$ assessment process. Ninety percent of dentists and 58 percent of dental hygienists did not have an established $\mathrm{AO} 4$ risk assessment plan/protocol. The majority of dentists and dental

Table 3: Dentists and dental hygienists AO4 assessment: Knowledge and practices.

\begin{tabular}{|c|c|c|c|c|}
\hline \multirow[b]{2}{*}{ Characteristic } & \multicolumn{2}{|c|}{ Dentists } & \multicolumn{2}{|c|}{ Dental Hygienists } \\
\hline & $\mathbf{N}$ & $\%$ & $\mathbf{N}$ & $\%$ \\
\hline \multicolumn{5}{|l|}{ Acceptable oral hygiene $(\mathrm{OH})$ standards } \\
\hline Poor OH unmanaged with professional periodontal maintenance therapy (PMT) & 4 & 8.2 & - & - \\
\hline Poor OH but managed with professional PMT & 2 & 4.1 & - & - \\
\hline Average $\mathrm{OH}$ with presence of biofilm and inflammation managed with PMT & 9 & 18.4 & - & - \\
\hline Excellent $\mathrm{OH}$ with minimal need of PMT to manage biofilm and inflammation & 9 & 18.4 & 1 & 2.9 \\
\hline Indicates does not see $\mathrm{AO} 4$ cases in their office & 10 & 20.4 & 22 & 62.9 \\
\hline \multicolumn{5}{|l|}{ Systemic contraindicated risk factors to $\mathrm{AO} 4$} \\
\hline Does not screen for systemic risk factors & - & - & 1 & 2.9 \\
\hline Indicates there are no contraindicated risk factors for AO4 treatment (Tx) & - & - & - & - \\
\hline Absence of all systemic diseases & 3 & 6.1 & 1 & 2.9 \\
\hline History of bisphosphonate use & 19 & 38.8 & 1 & 2.9 \\
\hline History of radiation therapy & 20 & 40.8 & 1 & 2.9 \\
\hline Uncontrolled diabetes & 25 & 51.0 & 2 & 5.7 \\
\hline Indicates does not know the contraindicating risk factors for $\mathrm{AO} 4$ & 6 & 12.2 & 18 & 51.4 \\
\hline Consider pregnancy and/or lactation a risk factor & 11 & 37.9 & 3 & 23.1 \\
\hline Does not consider pregnancy and/or lactation a risk factor & 18 & 62.1 & 10 & 76.9 \\
\hline \multicolumn{5}{|l|}{ Diagnostic oral assessment evaluation for appropriateness of AO4 } \\
\hline Take patient vital statistics & 19 & 38.8 & 3 & 8.6 \\
\hline Acquire complete and accurate medical and dental health history & 22 & 44.9 & 3 & 8.6 \\
\hline Review oral and nutritional habits & 16 & 32.7 & 2 & 5.7 \\
\hline Evaluate socioeconomic factors affecting treatment & 11 & 22.4 & 1 & 2.9 \\
\hline Evaluate the level of edentulism & 20 & 40.8 & 2 & 5.7 \\
\hline Acquire digital records/photos & 19 & 38.8 & 1 & 2.9 \\
\hline Take pre-operative models: Digital/articulated stone models & 20 & 40.8 & - & - \\
\hline Perform risk assessment & 22 & 44.9 & 1 & 2.9 \\
\hline Level of edentulism & 16 & 32.7 & - & - \\
\hline $\begin{array}{l}\text { I do not assess patients for } \mathrm{AO} 4 \text { treatment in my office; They present as a referral from } \\
\text { a specialist }\end{array}$ & 14 & 28.6 & 22 & 62.5 \\
\hline \multicolumn{5}{|l|}{ To what extent do you evaluate the level of edentulism? } \\
\hline Completely edentulous in both arches & 12 & 24.5 & - & - \\
\hline Completely edentulous in one arch and partially edentulous in the other arch & 12 & 24.5 & - & - \\
\hline $\begin{array}{l}\text { Partially edentulous in one or both arches but with the need of extraction of remaining } \\
\text { compromised teeth }\end{array}$ & 13 & 26.5 & - & - \\
\hline \multicolumn{5}{|l|}{ Has established AO4 treatment risk assessment form/protocol in office } \\
\hline Yes & 3 & 10 & 15 & 42.9 \\
\hline No & 27 & 90 & 20 & 57.1 \\
\hline Total & 30 & 100 & 35 & 100 \\
\hline \multicolumn{5}{|l|}{ Evaluation for risk assessment includes: } \\
\hline Bone quality & 20 & 40.8 & 2 & 5.7 \\
\hline Maxillary and mandibular profile in either healed or immediate extraction sites & 20 & 40.8 & 1 & 2.9 \\
\hline
\end{tabular}




\begin{tabular}{|c|c|c|c|c|}
\hline Parafunctional habits & 22 & 44.9 & 1 & 2.9 \\
\hline Tissue assessment & 22 & 44.9 & 2 & 5.7 \\
\hline Active infection & 22 & 44.9 & 2 & 5.7 \\
\hline Oral hygiene practices & 20 & 40.8 & 2 & 5.7 \\
\hline Systemic health & 23 & 46.9 & 2 & 5.7 \\
\hline History of radiation therapy & 23 & 46.9 & 1 & 2.9 \\
\hline Bisphosphonate use & 23 & 46.9 & 1 & 2.9 \\
\hline Tobacco - smoking & 23 & 46.9 & 2 & 5.7 \\
\hline Patient compliance & 20 & 40.8 & 2 & 5.7 \\
\hline Gender & 4 & 8.2 & 1 & 2.9 \\
\hline Does not screen/evaluate patients for $\mathrm{AO} 4$ in their office & 16 & 32.7 & 22 & 62.9 \\
\hline Total & 49 & 100 & 35 & 100 \\
\hline \multicolumn{5}{|c|}{ Performs screening for absence or presence of fenestration during tissue assessment during tissue assessment } \\
\hline Yes & 23 & 67.6 & 7 & 41.2 \\
\hline No & 10 & 29.4 & 10 & 58.8 \\
\hline Felt fenestrations and dehiscences do not affect treatment outcome of AO4 & 1 & 2.9 & - & - \\
\hline Total & 34 & 100 & 17 & 100 \\
\hline \multicolumn{5}{|l|}{ During periodontal assessment screens for: } \\
\hline Gingivitis & - & - & - & - \\
\hline Inactive periodontitis & 2 & 4.1 & - & - \\
\hline Active periodontitis & 11 & 22.4 & 10 & 47.6 \\
\hline Frenum attachment & 1 & 2.0 & 1 & 4.8 \\
\hline Soft tissue pathology & 4 & 8.2 & 3 & 14.3 \\
\hline Adequate keratinized tissue & 9 & 18.4 & 1 & 4.8 \\
\hline $\begin{array}{l}\text { None of the above; Periodontal disease and soft tissue pathology are not considered } \\
\text { risk factors in } \mathrm{AO} 4 \text { treatment }\end{array}$ & 4 & 8.2 & 6 & 28.6 \\
\hline Total & 31 & 100 & 21 & 100 \\
\hline \multicolumn{5}{|l|}{ Do you obtain digital records? } \\
\hline Yes & 32 & 76.2 & 20 & 74.1 \\
\hline No & 10 & 23.8 & 7 & 25.9 \\
\hline Total & 42 & 100 & 27 & 100 \\
\hline \multicolumn{5}{|l|}{ What do you use to acquire the data for your full-arch impressions? } \\
\hline Intraoral scanner & 7 & 14.3 & 1 & 2.9 \\
\hline Polyvinylsiloxane material & 27 & 55.1 & 2 & 5.7 \\
\hline Digitized software system & 3 & 6.1 & 3 & 8.6 \\
\hline Intraoral scanner for digital impressions & 3 & 6.1 & - & - \\
\hline I do not take full arch impressions for my $\mathrm{AO} 4$ cases & 3 & 6.1 & 3 & 8.6 \\
\hline I do not know anything about full-arch impressions & 3 & 6.1 & 14 & 40 \\
\hline \multirow[t]{2}{*}{ Total } & 49 & 100 & 35 & 100 \\
\hline & \multicolumn{4}{|c|}{ Dentists and dental hygienists } \\
\hline Characteristic & $\mathbf{N}$ & & $\%$ & \\
\hline \multicolumn{5}{|l|}{ What type of digital records do you obtain? } \\
\hline Clinical digital photographs & 42 & & 80.0 & \\
\hline Occlusal bite registration in maximum intercuspal position & 13 & & 25 & \\
\hline Full-arch impressions & 19 & & 36.5 & \\
\hline CBCT Scan with bite registration & 7 & & 13.5 & \\
\hline CBCT Scan without bite registration & 11 & & 21.2 & \\
\hline Orthopantomography & 26 & & 50 & \\
\hline Full mouth series of radiographs & 38 & & 73.1 & \\
\hline Intraoral scanner for digital impressions & 7 & & 13.5 & \\
\hline Total & 52 & & 100 & \\
\hline
\end{tabular}

hygienists indicated they do not take vital signs/statistics, complete an accurate medical and dental history, review oral/nutritional habits and socioeconomic factors, nor include a risk assessment (including systemic health, presence of active infection, bone quality, edentulism, tissue assessment, or history of radiation therapy and bisphosphonate use) in their $\mathrm{AO} 4$ oral assessment evaluation for their patients. Further, $32.7 \%$ of dentists and $62.9 \%$ of dental hygienists indicated they do not screen for $\mathrm{AO} 4$ in their dental practice. The majority of participants $(76.2 \%$ of dentists and $74.1 \%$ of dental hygienists) indicated utilizing diagnostic digital records for assessment of AO4 patients, and $13.5 \%$ of dentists and dental hygienists reported using an intraoral scanner for digital impressions. Fifty one percent of dentists agreed uncontrolled diabetes was a contraindication to AO4 treatment, but pregnancy and lactation were not (62.1\% of dentists and $76.9 \%$ of dental hygienists). Over 
Table 4: Differences between dentists and dental hygienists based on key variables ${ }^{\mathrm{a}}$.

\begin{tabular}{|c|c|c|c|c|c|}
\hline Variable & Pearson Chi Square & df & Significance & Phi & Cramer's V \\
\hline \multicolumn{6}{|l|}{ Knowledge } \\
\hline Received AO4 training & $7.304^{b}$ & 1 & 0.007 & -0.295 & \\
\hline Acceptable standards for patients recommended for $\mathrm{AO} 4$ & $24.697^{b}$ & 4 & 0.000 & & 0.658 \\
\hline Level of understanding of $\mathrm{AO} 4$ & 18.062 & 4 & 0.001 & & 0.573 \\
\hline \multicolumn{6}{|l|}{ Perceptions } \\
\hline Felt prepared to offer $\mathrm{AO} 4$ to patients & $1.574^{b}$ & 1 & 0.210 & 0.194 & \\
\hline Comfortable with maintenance therapy of patients with $\mathrm{AO} 4$ & $10.140^{\mathrm{b}}$ & 3 & 0.017 & & 0.47 \\
\hline Felt needed further training in $\mathrm{AO} 4$ & $1.792^{\mathrm{b}}$ & 1 & 0.181 & -0.189 & \\
\hline \multicolumn{6}{|l|}{ Self-reported practices } \\
\hline Participated in $\mathrm{AO} 4$ process & $4.057^{\mathrm{b}}$ & 1 & $0.044^{c}$ & 0.228 & \\
\hline Digital records & $0.040^{\mathrm{b}}$ & 1 & 0.842 & 0.024 & \\
\hline Are pregnancy and lactation risk factors for $\mathrm{AO} 4 ?$ & $0.891^{b}$ & 1 & 0.345 & 0.146 & \\
\hline Have an AO4 risk assessment form/protocol & $1.607^{b}$ & 1 & 0.205 & 0.189 & \\
\hline $\begin{array}{l}\text { Number of patients provided maintenance therapy who had } \\
\text { full-arch implant supported fixed prosthesis }\end{array}$ & $4.353^{\mathrm{b}}$ & 4 & 0.338 & & 0.307 \\
\hline \multicolumn{6}{|l|}{ Confidence } \\
\hline Confidence level with regard to $\mathrm{AO} 4$ process & $20.965^{b}$ & 4 & 0.000 & & 0.529 \\
\hline
\end{tabular}

aLevel of significance 0.05 ; ${ }^{\mathrm{b}} 1$ cells $(25.0 \%)$ have expected count less than 5 . The minimum expected count is 3.81 ; ' Due to lack of response (one participant responded to this question) this figure is calculated based on the continuity correction; Computed only for a $2 \times 2$ table with a measurement of 0.098 , and therefore is statistically insignificant.

half the dental hygienists $(51.4 \%)$ and a minimal number of dentists (12.2\%) indicated having no knowledge of the risk factors for AO4. Also noted, the majority of dentists $(46.7 \%)$ and dental hygienists $(66.7 \%)$ surveyed had not provided postoperative or maintenance therapy of AO4 patients. The majority of participants (57.2\% of dentists and $37.1 \%$ of dental hygienists) indicated referral of the surgical portion of $\mathrm{AO} 4$ treatment to an oral surgeon with a small percentage ( $14.3 \%$ of dentists and $11.4 \%$ of dental hygienists) providing no referral, but rather, opting to treat $\mathrm{AO} 4$ patients in their practice.

Pearson's chi-square analysis was used to compare participant responses on the variables of knowledge, perceptions, self-reported practices, and confidence in use of the $\mathrm{AO} 4$ implant process. A statistically significant difference was noted in the knowledge level for those who received $A O 4$ training $\left(X^{2}=7.30, d f=1, p=0.007\right)$ and for acceptable patient screening standards when recommending the $\mathrm{AO} 4$ process to patients $\left(X^{2}=24.69\right.$, $\mathrm{df}=1, \mathrm{p}=0.00)$. In addition, there was a statistically significant difference in the confidence levels between dentists and dental hygienists $\left(X^{2}=20.965, d f=4, p=\right.$ $0.000)$. No other variables demonstrated statistical significance. Table 4 depicts the differences between dentists and dental hygienists based on key variables.

Pearson's chi-square analysis was used to examine the differences between demographic variables and the knowledge level, perceptions, self-reported practices, and confidence levels of dentists and dental hygienists regarding the $\mathrm{AO} 4$ process. Statistically significant differences were noted for dentists when comparing degree level and responses to digital records $\left(X^{2}=3.95, \mathrm{df}\right.$ $=1, p=0.047), A O 4$ understanding $\left(X^{2}=12.096, d f=4\right.$, $p=0.017)$ and the need for further training $\left(X^{2}=15.148\right.$, $\mathrm{df}=1, \mathrm{p}=0.000$ ). No statistically significant differences were found between demographic variables and key variables studied among dental hygienists. No other variables tested demonstrated statistical significance. Analysis of any statistically significant relationship between the education level of dentists and dental hygienists and their self-reported practices, knowledge, perceptions, and confidence levels was not possible due to the variety of responses received, the small numbers of responses in some categories, and the number of blank responses for the category of educational level.

\section{Discussion}

Reported research on the efficacy of the $\mathrm{AO} 4$ process was completed in a controlled environment, in which all providers had extensive knowledge and years of experience with the risk assessment and systematic process for the treatment. A one hundred percent success rate was achieved [4,19-23]. While research is robust in support of a process that can deliver a $100 \%$ success rate, it is important to grasp the predominant factors contributing to this success. When providers have knowledge, experience, can repeat outcomes, and gain patient compliance, success can be achieved.

Oral implant processes such as the $\mathrm{AO} 4$ process involve multifaceted treatment plans and collaboration of all providers involved, as well as maintenance care to prevent peri-implant infection [35]. The success rate of dental implants has been reported in the literature as, on average, $98 \%$ [35]. However, minimal studies to date were completed to provide evidence as to whether or not this success rate was specifically affected by peri-implant maintenance therapy (PIMT) [35]. Globally, the incidence of peri-implant disease is reported as occurring at a frequency of up to 47\% [36-39]. Monj, Aranda, and Diaz, et al. completed a systematic review to assess the impact of maintenance therapy on the incidence of peri-implantitis [35]. The researchers found 
a significant link between PIMT frequency and impact on prevention of peri-implant disease. While supporting a three-prong approach to the implicating factors affecting implant failure or success rates (clinician, patient, and implant related dynamics), the researchers concluded that in addition to expert placement of oral implants, inclusion of an appropriate PIMT regimen had the potential to decrease implant failure rates [35]. In addition to maintenance, biologic and patient related factors, the knowledge and experience of the clinician can affect the outcome of the implant procedure.

In a 10-year retrospective study on the success rates of 50 full-arch maxillary and mandibular implant supported fixed prosthesis (297 implants placed) Ji, Kan, and Roe, et al. reported a cumulative success rate of $85.2 \%$ and an absolute success rate of $90.6 \%$ [40]. The results of this study suggested that the noted higher implant failure rates most likely were due to a history of bruxism (29.3\% of patients had a history of bruxism vs. $4.6 \%$ did not) and the limited experience of the clinician completing the treatment $(12.2 \%$ of surgeons had less than five years of experience and $2.4 \%$ had more). Further, a study by Lambert, Morris, and Ochi revealed dental implants placed by inexperienced clinicians failed twice as often as those placed by experienced clinicians; Supporting the conclusion that a "learning curve" existed with regard to success rates of dental implants [40]. In addition to level of experience, the complexity of the implant procedure can be a predominant predictor of implant success or failure rates $[40,41]$.

Statisticians anticipate the U.S. dental implant and prosthetic market will reach $\$ 6.4$ billion by the year 2018, with $10 \%$ of all practicing dentists reported as currently placing implants, and that number is said to be on the rise [42]. Due to the aging population and projected increase in demand for oral implants and prosthesis, systematic risk assessment will ultimately determine patient eligibility for advanced implant and prosthetic therapy [5]. Attention to advanced oral implant processes and interprofessional collaboration during implant treatment is considered another significant factor in the success of dental implants and prosthesis [5]. If the success rates of complex implant procedures are dependent on the knowledge and experience of the clinicians, the extent to which the protocols are followed, biologic patient factors determining the risk, and the maintenance therapy the patient receives; Then the components must be aligned in current treatment regimens [4,19-23,35,41,42]. Inexperienced dentists encounter higher failure rates while placing implants in immediate loading protocols for complex processes, like the $\mathrm{AO} 4$ process, than their more experienced counterparts $[35,43,44]$. Delivery of successful implant outcomes to patients is dependent on proficient skills, knowledge, and experience.

This exploratory study exposed a gap between the actual knowledge and skill level of the participants and their confidence levels with the AO4 process. The majority of dental hygienists reported they had never participated in and knew nothing of the $\mathrm{AO} 4$ process. Further, the majority of dentists exhibited minimal experience and knowledge with the AO4 process yet felt moderately to extremely confident in the $\mathrm{AO} 4$ process. Achieving high success rates with $\mathrm{AO} 4$ implant placement and outcomes is questionable if practitioners are neither educated nor experienced enough to accurately replicate the defined procedure. The results of this study revealed participants did not have the extensive knowledge and training in the $\mathrm{AO} 4$ process necessary to provide the reported $\mathrm{AO} 4$ success rates in their patient population. Most respondents reported no knowledge about the process, and no risk assessment protocol/procedure implemented in their offices. The results of this study have significant association with the conclusions reached by the comparable research $[35,40,43,44]$. Slightly more than one half of dentists who participated in the study reported confidence levels as moderately to extremely high, yet the majority reported their education did not effectively prepare them to provide $\mathrm{AO} 4$ and indicated a need for further $\mathrm{AO} 4$ training. Less than $20 \%$ of dentists reported participating in an $\mathrm{AO} 4$ case with one half reporting that during the case(s) they participated in, they were an observer vs. provider. Seventy percent of dentists reported never completing an AO4 case and only $14 \%$ of dentists had completed between one and ten cases. Data from this study indicated confidence levels disproportionate to the actual knowledge level and experience of the participants. There is a difference between knowledge and confidence. One can be confident, but without the appropriate knowledge and experience the patient may not receive the full benefits and success of the treatment modality. In order for the implant and prosthetic success rates to reach the $98 \%$ $100 \%$ levels, providers need a higher level of knowledge and expertise than demonstrated in this study.

\section{Limitations}

Limitations to this study must be considered. A self-designed questionnaire was utilized to conduct the study. This limitation was managed by conducting established validity and reliability measures. Another limitation was use of a convenience sample and the resultant low response rate. Those who responded to the survey were not representative of dentists and dental hygienists nationwide. Therefore, results cannot be generalized to the total population. The topic may not have been of particular interest to the sampled group. Research has shown topic relevance can contribute to a low response rate. In addition, some studies of survey response rates have demonstrated that web-based surveys tend to have lower response rates than mailed surveys, possibly reflecting participant's comfort level with computer technology or general preference for other survey methods [45-48].

Additional research concerning the variables of 
knowledge, attitudes, and practices of dentists and dental hygienists regarding oral implants, like the $\mathrm{AO} 4$ process, would provide more definitive conclusions. Particularly, addressing participant graduation date would provide insight to the timing of training with respect to the establishment of the $\mathrm{AO} 4$ process. Thus, having the potential to skew results as applied to education and training. Examining associations between variables and implant outcome and OHrQoL measures are important determinations of success. Comparisons should also be made with respect to implant procedure success rates and experience from Continuing education (CE) programs, as considerable variability exists within these programs. The protocols researched by experts in innovative prosthetic therapies direct specialty education, curriculum development, and availability to oral professionals $[4,5,19-23]$. The intent of specialty training is to "educate to a very high level and develop the art and science with an evidence-based approach" [5]. Thus, the future education of oral professionals is driven by the research and success rates of specific treatment modalities. Supplementary studies could illuminate the origination of this gap and if re-structured, could potentially address whether or not graduate and CE curriculum should be standardized and include more advanced oral implant procedures. Additionally, studies to determine whether or not advanced implant procedures, like $\mathrm{AO} 4$, should be contained to only graduate programs of study, where knowledge and expertise are more congruent, would prove beneficial. Professional education agencies such as the Commission on dental accreditation (CODA) have stringent standards with regard to oral specialties, but there do not appear to have similar standards for post graduate CE courses [49]. Concurrently, $84.7 \%$ of study participants reported receiving $\mathrm{AO} 4$ training from a CE course. Thus, future studies could aid in determination of the extent to which CE can be compared to current graduate level curriculum with regard to advanced implant therapies, provider knowledge and experience, and implant failure and success rates.

\section{Conclusion}

This study was designed to identify the knowledge level, perceptions, confidence levels, and self-reported practices of dentists and dental hygienists regarding the $\mathrm{AO} 4$ fixed prosthesis immediate load oral implant process. The results of this study revealed a gap in the knowledge level with regard to the training, the number of cases completed, the number of patients encountered while providing maintenance therapy, and the reported confidence levels of the practitioners. As oral professionals there is an ethical responsibility to ensure that education and knowledge level are congruent with experience of proffered treatment modalities and to ensure best practices can be provided to patients seeking advanced oral implant care.

\section{Sources of Support}

The author has no funding sources to report.

\section{Disclaimers/Disclosures}

The author has no conflicts of interest to disclose.

\section{References}

1. World Health Organization (2010) Framework for action on interprofessional education and collaborative practice, Geneva.

2. Ortman JM, Velkoff VA, Hogan H (2014) An aging nation: The older population in the United States. United States Census Bureau.

3. Douglass CW, Shih A, Ostry L (2002) Will there be a need for complete dentures in the United States in 2020? J Prosthet Dent 87: 5-8.

4. Babbush CA, Kutso GT, Borkloff J (2011) The all-on-four immediate function treatment concept with nobel active implants: A retrospective study. J Oral Implant 37: 431-445.

5. Clem DS (2014) Dental implant's future: The need for a team approach. Compend Contin Educ Dent 35: 608-609.

6. Montero J, Castillo-Oyagu R, Lynch CD, Albaladejo A, Castano A (2013) Self-perceived changes in oral health-related quality of life after receiving different types of conventional prosthetic treatments: A cohort follow-up study. J Dent 41: 493-503.

7. Allen F, Locker D (2002) A modified short version of the oral health impact profile for assessing health-related quality of life in edentulous adults. Int J Prosthodont 15: 446-450.

8. World Health Organization (2011) Global health and aging, Geneva.

9. Kikutani T, Yoshida M, Enoki H, Yamashita Y, Akifusa S, et al. (2013) Epidemiology, clinical practice, and health: Relationship between nutrition status and dental occlusion in community-dwelling frail elderly people. Geriatr Gerontol Intl 13: 50-54.

10. Kaiser M, Bandinelli S, Lunenfeld B (2010) Frailty and the role of nutrition in older people: A review of the current literature. Acta Biomed 81: 37-45.

11. Walls A (2014) Developing pathways for oral care in elders: Challenges in care for the dentate the subject? Gerodontology 31: 25-30.

12. Cosola S, Marconcini S, Giammarinaro E, Poli GL, Covani $U$, et al. (2018) Oral health-related quality of life and clinical outcomes of immediately or delayed loaded implants in the rehabilitation of edentulous jaws: A retrospective comparative study. Minerva Stomatol 67: 189-195.

13. Papaspyridakos P, Chen CJ, Chuang SK, Weber HP (2014) Implant loading protocols for edentulous patients with fixed prostheses: A systematic review and meta-analysis. Int J Oral Maxillofac Implants 29: 256-270.

14. Papaspyridakos P, Lal K (2010) Immediate loading of the maxilla with prefabricated interim prosthesis using interactive planning software and CAD/CAM rehabilitation with definitive zirconia prosthesis: 2-Year clinical follow-up. J Esthet Restor Dent 22: 223-232.

15. Pikos MA, Magyar CW, Llop DR (2015) Guided full-arch immediate-function treatment modality for the edentulous and terminal dentition patient. Compend Contin Edu Dent 36: 119-126.

16. de Avila ÉD, de Molon RS, de Assis Mollo F Jr, de Barros LA, Capelozza Filho L, et al. (2012) Multidisciplinary approach for the aesthetic treatment of maxillary lateral in- 
cisors agenesis: Thinking about implants? Oral Surg Ora Med Oral Pathol Oral Radiol 114: 22-28.

17. Felisati G, Saibene AM, Pipolo C, Mandelli F, Testori T (2014) Implantology and otorhinolaryngology team-up to solve a complicated case. Implant Dent 23: 617-621.

18. Dyer TA, Owens J, Robinson PG (2013) What matters to patients when their care is delegated to dental therapists? Br Dent J 214: E17.

19. Malo P, de Auaujo-Nobre M, Lopes A, Francischone C, Rigolizzo M (2012) All-on-4 immediate-function concept for completely edentulous maxillae: A clinical report on the medium (3 years) and long-term (5 years) outcomes. Clin Implant Dent Relat Res 14: e139-e150.

20. Malo P (2013) Nobel biocare: The evolution of the all-on-4® treatment concept.

21. Malo P, Rangert B, Nobre M (2003) "All-on-Four" immediate-function concept with Branemark System implants for completely edentulous mandibles: A retrospective clinical study. Clin Implant Dent Relat Res 5: 2-9.

22. Malo P, Araujo-Nobre M, Lopes A, Ferro A, Gravito I (2015) All-on-4® treatment concept for the rehabilitation of the completely edentulous mandible: A 7-year clinical and 5-year radiographic retrospective case series with risk assessment for implant failure and marginal bone level. Clin Implant Dent Relat Res 17: 531-541.

23. Nobel biocare (2017) All-on-4: The efficient treatment concept with immediate loading.

24. Marra R, Acocella A, Rispoli A, Sacco R, Ganz SD, et al. (2013) Full-mouth rehabilitation with immediate loading of implants inserted with computer-guided flap-less surgery: A 3-year multicenter clinical evaluation with oral health impact profile. Implant Dent 22: 444-452.

25. Di P, Lin Y, Li JH, Luo J, Qiu LX, et al. (2013) The all-onfour implant therapy protocol in the management of edentulous Chinese patients. Int J Prosthodont 26: 509-516.

26. Krennmair S, Seemann R, Weinlander M, Krennmair G, Peihslinger E (2014) Immediately loaded distally cantilevered fixed mandibular prostheses supported by four implants placed in both in fresh extraction and healed sites: 2-year results from a prospective study. Eur J Oral Implantol 7: 173184.

27. Penarrocha-Diago MA, Maestre-Ferrin L, Demarchi CL, Penarrocha-Oltra D, PenarrochaDiago M (2011) Immediate versus nonimmediate placement of implants for full-arch fixed restorations: A preliminary study. J Oral Maxillo Surg 69: 154-159.

28. Bylaws and code of ethics (2016) American Dental Hygienist's Association.

29. Genco RJ, Genco FD (2014) Common risk factors in the management of periodontal and associated systemic diseases: The dental setting and interprofessional collaboration. J Evid Based Dent Pract 14: 4-16.

30. Braun PA, Kahl S, Ellison MC, Ling S, Widmer-Racich K, et al. (2013) Feasibility of collocating dental hygienists into medical practices. J Public Health Dent 73: 187-194.

31. Hein C (2009) Translating evidence of oral-systemic relationships into models of interprofessional collaboration. J Dent Hyg 83: 188-189.

32. Zwarenstein M, Goldman J, Reeves S (2009) Interprofessional collaboration: Effects of practice-based interventions on professional practice and healthcare outcomes. Cochrane Database Syst Rev.
33. Oregon Board of Dentistry. Regulations. Portland.

34. Polit DF, Beck CT (2006) The content validity index: Are you sure you know what's being reported? Critique and recommendations Res Nurs Health 29: 489-497.

35. Monje A, Aranda L, Diaz KT, Alarcon MA, Bagramian RA, et al. (2016) Impact of maintenance therapy for the prevention of peri-implant diseases: A systematic review and meta-analysis. J Dent Res 95: 372-379.

36. Zitzmann NU, Berglundh T (2008) Definition and prevalence of peri-implant diseases. J Clin Periodontol 35: 286291.

37. Atieh MA, Alsabeeha NH, Faggion CM Jr, Duncan WJ (2013) The frequency of peri-implant diseases: A systematic review and meta-analysis. J Periodontol 84: 1586-1598.

38. Derks J, Tomasi C (2015) Peri-implant health and disease. A systematic review of current epidemiology. J Clin Periodontol 42: 158-171.

39. Jepsen S, Berglundh T, Genco R, Aass AM, Demirel K, et al. (2015) Primary prevention of peri-implantitis: Managing peri-implant mucositis. J Clin Periodontol 42: 152-157.

40. Ji TJ, Kan JY, Rungcharassaeng K, Roe P, Lozada JL (2012) Immediate loading of maxillary and mandibular implant-supported fixed complete dentures: A 1- to 10-year retrospective study. J Oral Implantol 38: 469-476.

41. Porter JA, Fraunhofer JA von (2005) Success or failure of dental implants? A literature review with treatment considerations. Gen Dent 53: 423-432.

42. American Academy of implant dentistry. Dental implants facts and figures.

43. Lambert PM, Morris HF, Ochi S (1997) Positive effect of surgical experience with implants on second-stage implant survival. J Oral Maxillofac Surg 55: 12-18.

44. Barone A, Toti P, Marconcini S, Derchi G, Saverio M, et al. (2016) Esthetic outcome of implants placed in fresh extraction sockets by clinicians with or without experience: A medium-term retrospective evaluation. Int J Oral Maxillofac Implants 31: 1397-1406.

45. Cook C, Heath F, Thompson RL (2000) A meta-analysis of response rates in web- or internet-based surveys. Educ Psychol Meas 60: 821-836.

46. Boyer KK, Olson JR, Jackson EC (2001) Electronic surveys: Advantages and disadvantages over traditional print surveys. Decision Line 4-7.

47. Manfreda KL, Bosnjak M, Berzelak J, Haas I, Vehovar V (2008) Web surveys versus other survey modes. Int J Market Res 50: 79-104.

48. Hardigan PC, Succar CT, Fleisher JM (2012) An analysis of response rate and economic costs between mail and webbased surveys among practicing dentists: A randomized trial. J Community Health 37 : 383-394.

49. Commission on Dental Accreditation (2016) Current accreditation standards, Chicago. 